\title{
Teaching digital holography through an interface in Java
}

Ma. Araiza-Esquivel, Pilar C. Godina, Alfonso LópezMartínez, Carlos Olvera, Santiago Villagrana Barraza, et al.

Ma. Araiza-Esquivel, Pilar C. Godina, Alfonso López-Martínez, Carlos Olvera, Santiago Villagrana Barraza, Diana Ortiz-Daz, "Teaching digital holography through an interface in Java," Proc. SPIE 11143, Fifteenth Conference on Education and Training in Optics and Photonics: ETOP 2019, 111430E (2 July 2019); doi: 10.1117/12.2520784 


\title{
Teaching digital holography through an interface in Java
}

\author{
Ma. Araiza-Esquivel ${ }^{\mathrm{a}}$, Pilar C. Godina ${ }^{\mathrm{a}}$, Alfonso López-Martínez ${ }^{\mathrm{a}}$, Carlos Olvera ${ }^{\mathrm{a}}$, Santiago \\ Villagrana Barraza ${ }^{a}$, and Diana Ortiz-Daz ${ }^{\mathrm{a}}$ \\ a Unidad Académica de Ingeniería Eléctrica, Universidad Autónoma de Zacatecas (UAZ), \\ Zacatecas 98000, México
}

\begin{abstract}
We present an interactive simulation software to help to teach and learn the holography concept. The education interface was developed in the Java platform. The Holographic Interface is a computer assisted learning that can be used in classrooms or for distance education. The binary holograms are numerically generated and reconstructed in the virtual optical laboratory. Several procedures are shown. The interface has been implemented with different options such as addition, subtraction, multiplexing and some properties of holography. Moreover, the software was designed to simultaneously visualize the $2 \mathrm{D}$ object, the generated hologram and the recovered image. We have taken into account the students' suggestions in this version in Java.
\end{abstract}

Keywords: Computer Generated Holography, Software, Education

\section{INTRODUCTION}

Holography is an important branch of optics. A sub-branch of Holography is the Computer-Cenerated Hologram (CGH) that has been studied and analyzed. The binary CGH has multiple applications. ${ }^{1-5}$

The detour-phases binary CGH uses the width and position to codify the amplitude and phase of a light wavefront, respectively. The generated opaque regions in a set of cells are spatially distributed in the hologram. ${ }^{2}$ Detour CGHs are simple techniques, they work with two-dimensional images, and the generated pattern can be reliable storage format. Some function can be implemented using binary holograms, such as addition, subtraction, multiplexing, or shift among others may be visually demonstrated. ${ }^{6}$

In this paper, we show an improvement on the previous version of the holographic interface (HI) ${ }^{6}{ }^{\text {The }}$ interface is developed on the Java platform and has four more function windows. The simulation software was designed to work with the detour type binary hologram. The HI is conceived to help students to understand and learn basic digital holography concepts. The numerical hologram can be recorded and reconstructed in the interface where the object, the hologram and the reconstructed image of the object are showed. Each of the elements can have different magnifications.

This paper is organized as follows. In section 2, the technique that has been implemented in the interface to encode computer-generated holograms is shown. The holographic interface structure is shown in section 3, and the available fuctions are described. In section 4 , simulation results are presented. Finally, in section 5 , the conclusions are shown.

\section{TECHNIQUE: COMPUTER GENERATED HOLOGRAM (CGH)}

Figure 1 shows one of the $N \times N$ cells of a CGH, of size $\delta \nu \times \delta \nu$. The cell is the minimal element in a hologram, defined between $N / 2$ and $N / 2$, including the hologram information as shown. The rectangle inside the cell is defined with two free parameters width $W_{m n}$, and lateral position $P_{m n}$, to codify the discrete Fourier Transform (DFT) of the object amplitude $A_{m n}$, and phase $\phi_{m n}$, respectively. The $m$ and $n$ indexes correspond to the vertical and horizontal position in the Fourier space, respectively.

At the hologram plane $\left(\nu_{x}, \nu_{y}\right)$, the binary amplitude transmittance of a cell is $[4,5]$ :

Further author information: (Send correspondence to M.A.E. )

MAE: E-mail: araizama@uaz.edu.mx, Telephone: 1524929256690

Fifteenth Conference on Education and Training in Optics and Photonics: ETOP 2019, edited by

Anne-Sophie Poulin-Girard, Joseph A. Shaw, Proc. of SPIE Vol. 11143, 111430E - (C) 2019

SPIE, ICO, IEEE, OSA · CCC code: 0277-786X/19/\$18 · doi: 10.1117/12.2520784

Proc. of SPIE Vol. 11143 111430E-1 


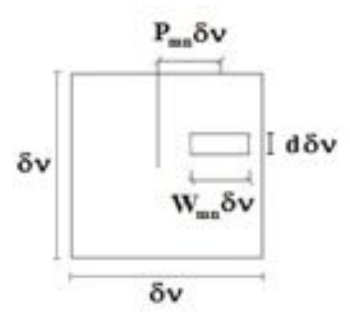

Figure 1. Cell, minimal element of a CGH.

$$
H\left(\nu_{x}, \nu_{y}\right)=\left[\frac{\nu_{x}-\left(n+P_{m, n}\right) \delta \nu}{W_{m, n} \delta \nu}\right]\left[\frac{\nu_{y}-m \delta \nu}{\delta \nu}\right]
$$

where

$$
\begin{array}{r}
W_{m, n}=\frac{\sin ^{-1} A_{m, n}}{\pi}, \\
P_{m, n}=\frac{\varphi_{m, n}}{2 \pi} .
\end{array}
$$

A complex two-dimensional optical object is defined with $u(x, y)=|u(x, y)| \exp [i \phi(x, y)]$ and its DFT is $U=A_{m, n} \exp \left[i \varphi_{m, n}\right]$. After the free parameters have been calculated, the simulated Fourier hologram is graphed as a matrix of $N \times N$ size.

Figure 2 shows an optical setup to reconstruct the image as an experimental way. On the other hand, by appying the IFT, the reconstructed image can be calculated numerically as a simulated procedure.

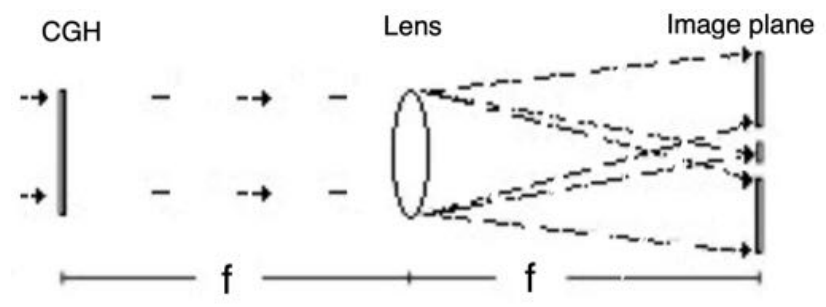

Figure 2. Optical setup for experimental reconstruction.

At the image plane $\left(x^{\prime}, y^{\prime}\right)$, the Inverse Fourier Transform (IFT) of $H\left(\nu_{x}, \nu_{y}\right)$ could be written as a complex function:

$$
\begin{array}{rc}
h\left(x^{\prime}, y^{\prime}\right) & =\quad \iint H\left(\nu_{x}, \nu_{y}\right) \exp \left[2 \pi i\left(x^{\prime} \nu_{x}+y^{\prime} \nu_{y}\right)\right] d \nu_{x} \nu_{y} \\
& =\sum_{m, n} W_{m, n} \operatorname{sinc}\left(x^{\prime} W_{m, n} \delta \nu\right) \exp \left[2 \pi i x^{\prime}\left(n+P_{m, n}\right) \delta \nu\right]
\end{array}
$$

where $h\left(x^{\prime}, y^{\prime}\right)$ represents again the original image $u(x, y)$, appearing in the first diffraction order.

The experimental procedure is as follows: the discrete cells pattern is plotted and photographically reduced. Diffracting transparency is obtained when coherent light illuminates the reduced hologram (the transparency). The twin conjugated images resembling the original object, $u(x, y)$ appear at the output plane as shown in Fig. 2 .

The simulated procedure is as follow: the reconstructed image is recovered from the the hologram numerically calculated, that is the inverse Fourier Transform (IFT) of the CGH is applied. At the image plane two conjugate images resembling $u(x, y)$, can be displayed on the screen of a display. 


\section{HOLOGRAPHIC INTERFACE.}

In this work, we present a software to simulate binary detour type computer-generated holograms. We can make and reconstruct a hologram to perform some of the mathematical operations. In addition, the object, the hologram and the entire image plane can be seen and magnified together or separately. Fig. 3 shows the main window of the interface, that was implemented using Java. The first version has five functions, but this new version has eight functions. The Holographic Interface main window shows buttons. Each or these buttons sends you a secondary window where different functions are implemented. This main window, shown in Fig. 3, contains the links to the specific function windows: creating a hologram, adding or subtracting a couple of holograms, multiplexing them, orthogonal multiplexing, different frequency, dimensional object, and a holography property. We can find four different forms in each secondary window to manipulate a hologram: choose the object or objects, modify the cell size, or size of an image, and reconstruct an image. Moreover, at the secondary window, we can select one of the sub figures and open it in a third window, where the figure is magnified.

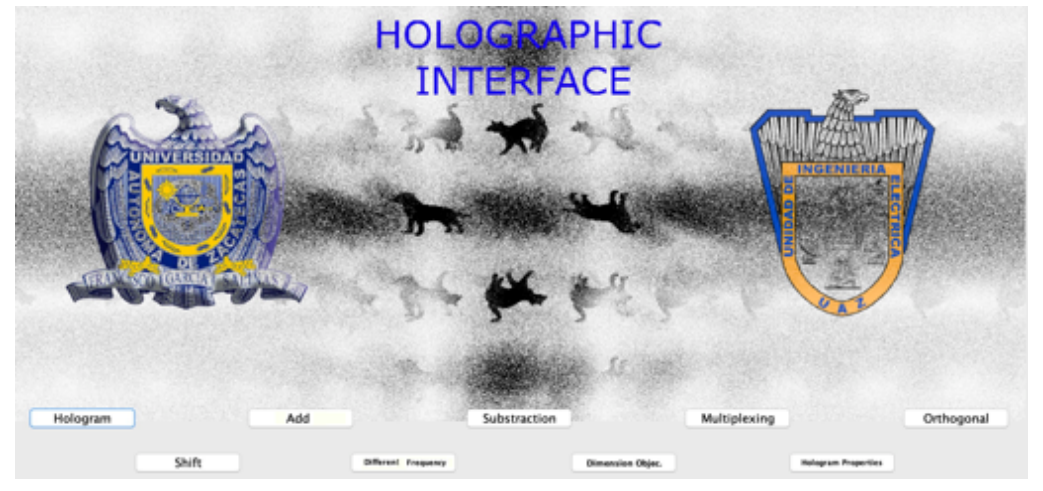

Figure 3. Holographic interface for generating and reconstructing numerical holograms.

The program is a highly user-friendly interface showing different type of buttons as open, save, help, exit and remove phase diffuser. On the other hand, the interface shows error messages when the program cannot be fulfilled, as shown in Fig. 4.
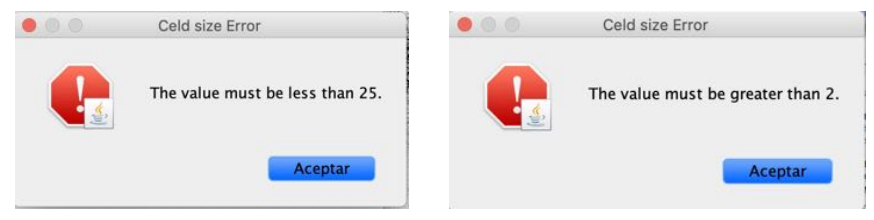

Figure 4. Error messages.

The general procedure for an implemented function in a function window is explained as follows. See Fig. 5. First, opening a two-dimensional digital image, see Fig. 5a). Then, a button for generate a hologram is activated, see Figure 5b). The active button is pressed and a few seconds later a new temporal window appears, asking the cell size, Fig. 5c). The number of pixel in the image is the same as the number of cells of the hologram. A hologram image, the image plane, and a magnified reconstructed image of first order are displayed in some sub-windows in the secondary window of every specific function, see Fig. 5d). The two-dimensional image is codified in a computer-generated hologram of $N \times N$ cells. The Inverse Fourier Transform (IFT) is computed and the reconstructed image of one or more objects is recovered in the image plane. ${ }^{6,7}$

\section{FUNCTIONS WINDOW.}

The purpose of the functions implemented in the interface are explained in the following paragraphs. 

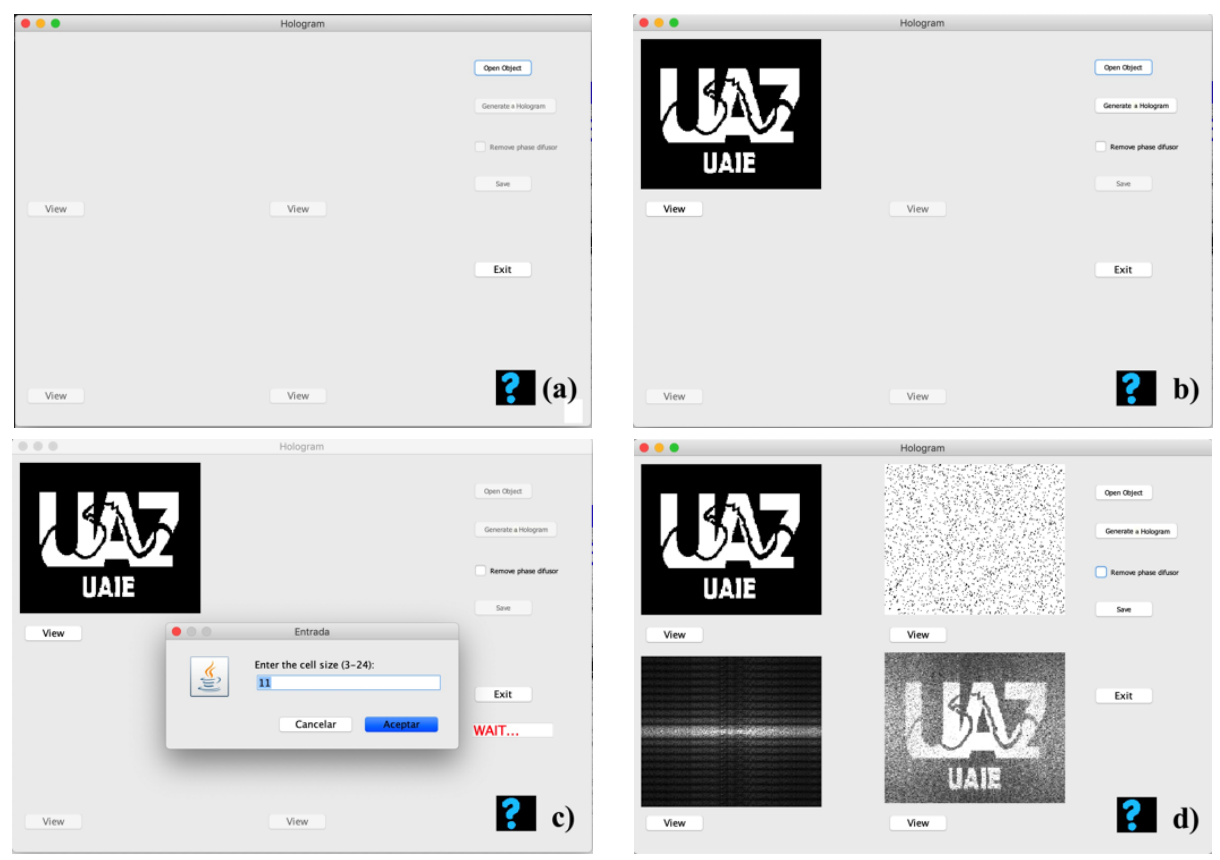

Figure 5. The General procedure to generate a hologram.

\section{Hologram.}

A hologram is made in the first option of the interface. Fig. 5 shows the general procedure for generate a hologram. ${ }^{6}$ A digital object is loaded and a hologram is made by pushing the generate a hologram button. Each sub-window can be selected, amplified or saved.

\section{Add.}

Figure $6 \mathrm{~b}$ ) shows the addition of two objects. ${ }^{6}$ The algebraic addition of the discrete Fourier Transform (DFT) of two digital objects (Fig. 6a)).

$$
\mathcal{F}\left\{A_{1} \exp \left(i \phi_{1}\right)\right\}+\mathcal{F}\left\{A_{2} \exp \left(i \phi_{2}\right)\right\}
$$

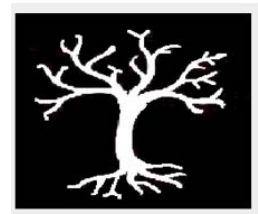

view

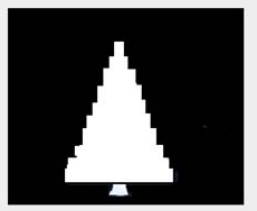

a)
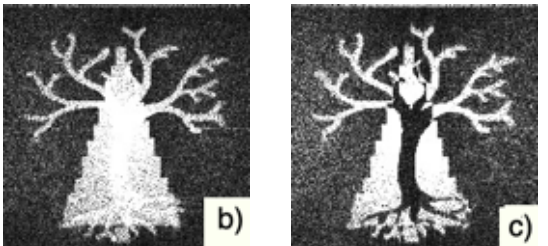

Figure 6. Algebraically a) Objects, b) Addition, and c) Subtraction.

\section{Subtraction.}

Figure 6c) is the subtraction, it is a specially case of sum, the second object is encoded with a global phase delay of $\pi$, as follows: ${ }^{6}$

$$
\mathcal{F}\left\{A_{1} \exp \left(i \phi_{1}\right)\right\}+\mathcal{F}\left\{-A_{2} \exp \left(i \phi_{2}\right)\right\} .
$$

\section{Multiplexing.}

Figure 7 shows the simulated multichannel technique, codifying three objects, each object is encoded in a different subcell, as it is explained in Ref. 7 . 


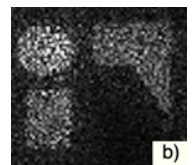

Figure 7. Multiplexing. Three different objects were encoded in the same hologram.

\section{Orthogonal.}

Figure 8 shows the simplified orthogonal multichannel technique. Two different objects are encoded in orthogonal directions. Details about the multiplexing method are reported in Ref. 8.

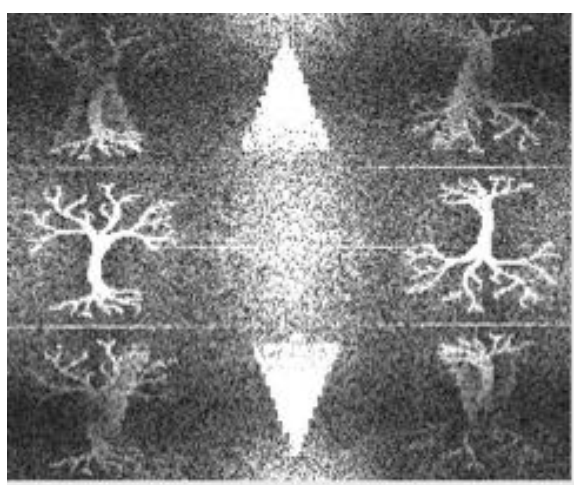

Figure 8. Orthogonal multiplexing: Two object encoded in orthogonal coordinates.

\section{Shift.}

The shift theorem ${ }^{9,10}$ is applied in the function window. Fig. 9 shows the reconstructed image with a shift in $\mathrm{Y}$ coordinate. The shift theorem is:

$$
H\left(\nu_{x}, \nu_{y}\right) \exp \left[i\left(\nu_{x} \varpi_{0}+\nu_{y} \eta_{0}\right)\right] \longleftrightarrow^{F} h\left(x^{\prime}-\varpi_{0}, y^{\prime}-\eta_{0}\right)
$$

where $\varpi_{0}$ and $\eta_{0}$ are constants.

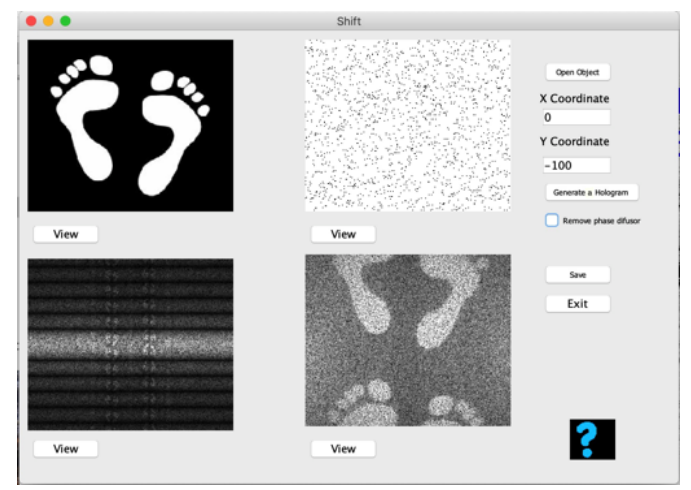

Figure 9. Shift function.

\section{Frequency.}

In this function window, two overlapping holograms are generated. The objects and holograms have the same number of pixels and of cells, but different cell size. The objective is to show the recovered images when cell size changes. See Fig. 10. 


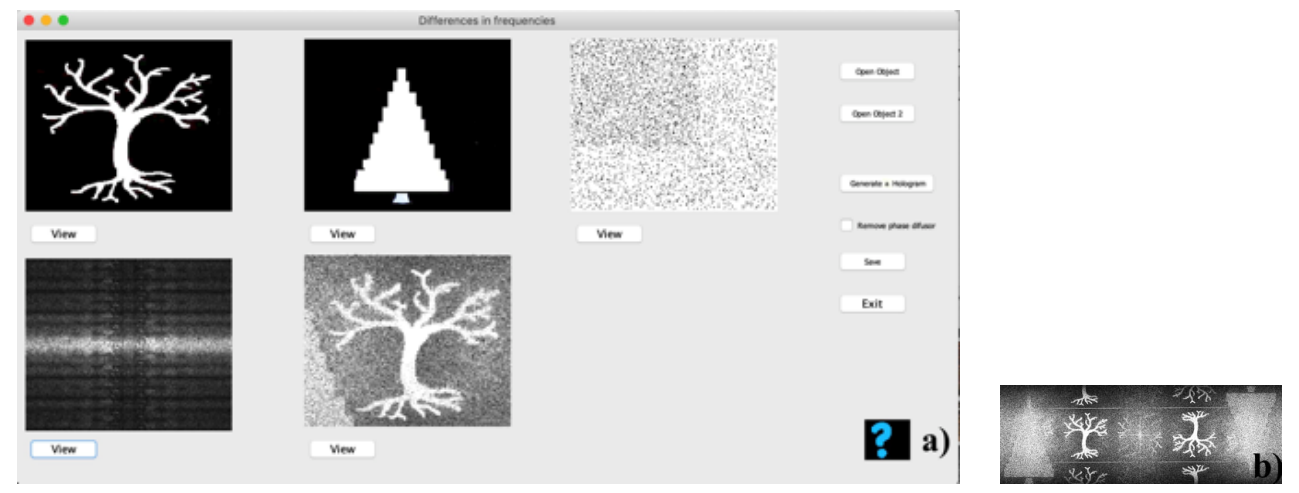

Figure 10. a) Overlapping two different holograms with different cell size, b) amplification of the image plane ( zero, +1, -1 orders).

\section{Dimension of objects.}

In this function window, two objects have a different number of pixels, one of the objects is bigger than the second. Their respective holograms are generated using the same cell size. Fig. 11 shows how the resolution of the reconstructed images increases more and more in relation to the size of the object. There is a relationship between the cell size and the size of the image. The first reconstructed image has more resolution than the second.

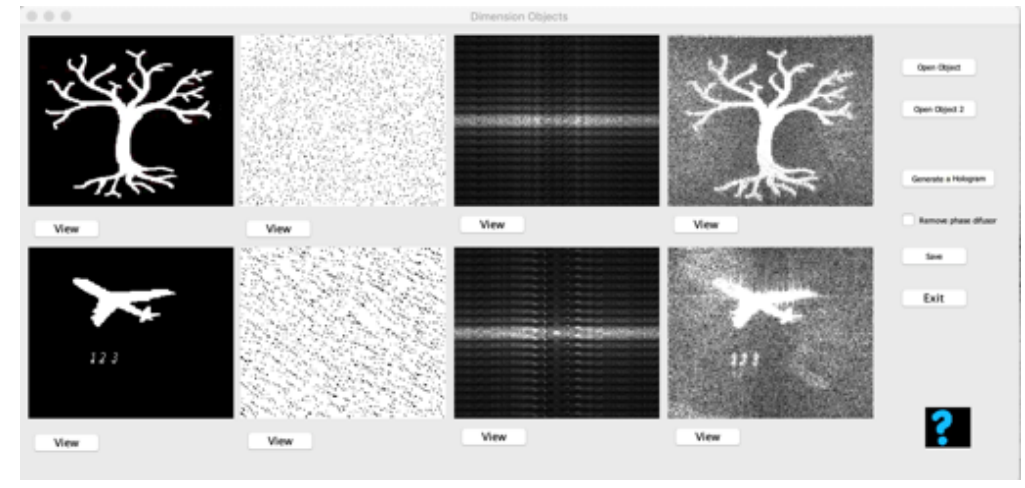

Figure 11. Two objects of different number of pixels are encoded in their hologram, the same cell size was used.

\section{Holograms property.}

In a simulation process or in analogy way, when we lost part of the hologram, it is valid to recover the information encoded in a hologram even if we only have a little section of it. In this function window, a section of the hologram that will be reconstructed is selected. We can reconstruct the original image with less resolution as, it can be shown in Fig. 12.

\section{CONCLUSIONS.}

We come to the conclusion that, since the holographic interface is a visual tool, it can be a first approach to understanding the basic concepts of holography in an easier way without having to go to a laboratory..

The educational holographic interface is based on the detour type hologram and it is designed to learn a little more about holography. It can be possible to visualize the numerical processes of a hologram generation and reconstruction. 


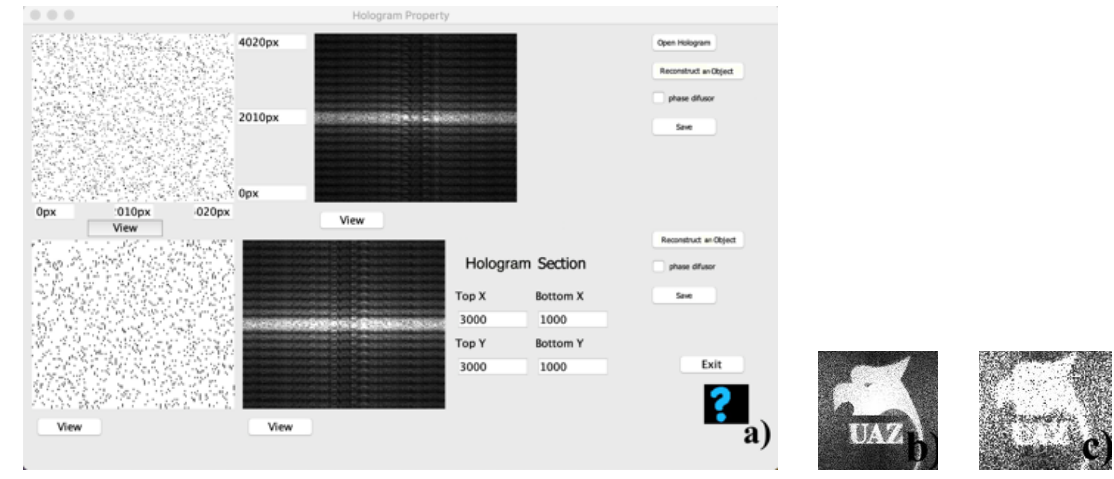

Figure 12. Reconstructed image of: a) $N \times N$ hologram, b) section of hologram .

The simulation program was designed with the intention of being a holography educational tool for students with a knowledge of optics. The software introduces some basic concepts to understand the holography topic.

The interface developed in the Java platform has four more function windows than the previous version, and each function window has help to guide the student. The simulated results agree with the experiment result reported in Refs. 7 to 8 and 10.

\section{Acknowledgement.}

The authors acknowledge Enrique Tajahuerce, Lluís Martínez-León, Carlos A. Guerrero, Javier González Trejo, Manuel López, I. Barrón and Job Martínez for their useful suggestions and encouraging comments, by the time this project was suggested.

\section{REFERENCES}

[1] Huang, Z., Marks, D. L., and Smith, D. R., "Out-of-plane computer-generated multicolor waveguide holography," Optica 6(2), 119-124 (2019).

[2] Brown, B. R. and Lohmann, A. W., "Complex spatial filtering with binary masks," Appl. Opt. 5(6), 967-969 (1966).

[3] Lee, W.-H., "Binary synthetic holograms," Applied optics 13(7), 1677-1682 (1974).

[4] Lee, W.-H., "III Computer-Generated Holograms: Techniques and Applications," in [Progress in Optics], Wolf, E., ed., 16, 119-232, Elsevier (1978).

[5] Bartelt, H., "Space multiplexing with computer holograms," Optics Communications 23(2), 203-206 (1977).

[6] Araiza-Esquivel, M., Tajahuerce, E., Martínez-León, L., González-Ramírez, E., Villa-Hernández, J., OlveraOlvera, C., Guel-Sandoval, S., and Tsonchev, R., "Learning digital holography through an interactive virtual lab: The holographic interface," in [EDULEARN11 Proceedings], 3rd International Conference on Education and New Learning Technologies, 4774-4781, IATED (4-6 July, 2011 2011).

[7] Araiza-Esquivel, M., Guel-Sandoval, S., and Ojeda-Castaeda, J., "Multichannel image storage with image processing capabilities," Optics Communications 230(1-3), 131-135 (2004).

[8] Araiza-Esquivel, M. and Guel-Sandoval, S., "Two-channel computer-generated holograms: a simplified method," Optics and Lasers in Engineering 39(5), 629 - 634 (2003).

[9] Goodman, J. W., [Introduction to Fourier Optics], Roberts ans Company Publishers, USA, third ed. (2005).

[10] Esquivel, M. A. A., Guel-Sandoval, S., and Lastras, L., "Desplazamientos de imágenes con hologramas generados por computadora," 46(3), 300-303 (2000). 